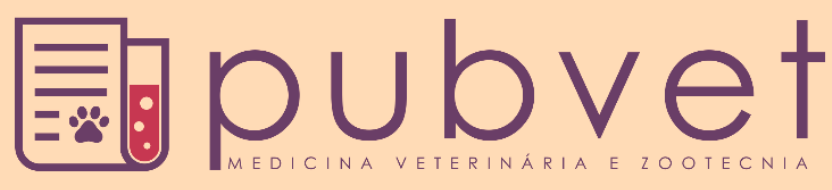

https://doi.org/10.31533/pubvet.v15n09a914.1-4

\title{
Gastrosquise e toracosquise em um canino
}

\author{
Ana Clara de França Silva ${ }^{1}$, Lylian Karlla Gomes Medeiros $^{2}{ }^{\circ}$, Gracineide da Costa Felipe ${ }^{2}$, $^{\circ}$, \\ Luan Aragão Rodrigues ${ }^{3}{ }^{*}$, Fabrícia Geovania Fernandes Filgueira ${ }^{4}$, Marcelo Jorge \\ Cavalcanti de $\mathrm{Sá}^{5}{ }^{\circ}$, Pedro Isidro da Nobrega Neto ${ }^{6}{ }^{\circ}$ \\ ${ }^{1}$ Professora substituta do Instituto Federal da Paraíba, Hospital Veterinário Adílio Santos de Azevedo (HV-ASA) - PB Brasil. \\ ${ }^{2}$ Doutoranda pelo Programa de Medicina Veterinária da Universidade Federal de Campina Grande (UFCG), Patos - PB Brasil. \\ ${ }^{3}$ Aluno do Curso de Especialização em Medicina Veterinária do Instituto Federal da Paraíba, HV-ASA - PB Brasil. \\ ${ }^{4}$ Médica Veterinária do Instituto Federal da Paraíba, HV-ASA - PB Brasil. \\ ${ }^{5}$ Professor doutor do curso de Medicina Veterinária da UFCG, Patos - PB Brasil. \\ ${ }^{6}$ Professor doutor do curso de Medicina Veterinária da Universidade Federal de Campina Grande, Patos - PB Brasil. \\ *Autor para correspondência, E-mail: luan.veterinaria@gmail.com
}

Resumo. Objetivou-se neste trabalho descrever uma malformação congênita observada em neonato canino atendido em um Hospital Veterinário Escola. $\mathrm{O}$ feto apresentava alterações macroscópicas de gastrosquise e toracosquise. Devido ao grande defeito anatômico observado no neonato canino do presente estudo e as escassas informações na literatura de como proceder com essas malformações congênitas na medicina veterinária bem como a falha no tratamento de suporte neonatal, optou-se pela eutanásia do feto. Por se tratar de uma malformação congênita, a gastrosquise e toracosquise apresentam tratamento limitado, sugerindo a necessidade de estudos sobre o desenvolvimento embrionário, a eficiência de técnicas cirúrgicas aplicáveis e tratamento de suporte neonatal adequado, a fim de garantir um bom prognóstico para os pacientes portadores.

Palavras chave: Congênita, cirurgia, neonatologia

\section{Gastroschisis and toracoschisis in a canine}

Abstract. The objective of this study was to describe a congenital malformation observed in a neonate dog treated at a Veterinary School Hospital. The fetus presented macroscopic alterations of gastroschisis and thoracosquise. Due to the great anatomic defect observed in the canine neonate of the present study and the scarce information in the literature on how to proceed with these congenital malformations in veterinary medicine as well as the failure in the treatment of neonatal support, fetal euthanasia was chosen. Because it is a congenital malformation, gastroschisis and thoracoschisis present limited treatment, suggesting the need for studies on embryonic development, the efficiency of applicable surgical techniques and the treatment of adequate neonatal support in order to guarantee a good prognosis for the patients.

Keywords: Anomalies, neonatology, surgery

\section{Gastrosquisis y toracosquis en un canino}

Resumen. El objetivo de este estudio fue describir una malformación congénita observada en un neonato canino tratado en Hospital Escuela Veterinaria. El feto tuvo cambios macroscópicos en gastrosquisis y toracosquisis. Debido al gran defecto anatómico observado en el canino neonato del presente estudio y la escasa información en la literatura sobre cómo proceder con estas malformaciones congénitas en medicina veterinaria, así como el fracaso en el tratamiento del soporte neonatal, optamos por la eutanasia del feto. Al tratarse de una malformación congénita, la gastrosquisis y la toracosisis tienen un 
tratamiento limitado, lo que sugiere la necesidad de estudios sobre el desarrollo embrionario, la eficacia de las técnicas quirúrgicas aplicables y el tratamiento de un adecuado soporte neonatal, con el fin de asegurar un buen pronóstico a los pacientes.

Palabras clave: Congénito, cirugía, neonatología

\section{Introdução}

Os defeitos congênitos encontrados nos neonatos correspondem a alterações nas proporções corporais, cabeça e face, abdome, tórax e coluna vertebral, membros e cauda, órgãos genitais e ânus (Jericó et al., 2015). As causas mais comuns desses defeitos podem ser maternais, gestacionais, ambientais, genéticas e por fatores infecciosos (Little, 2011).

A gastrosquise é um defeito na parede abdominal, que ocorre devido uma falha no fechamento da musculatura reto do abdômen durante o período fetal. Essa falha causa a saída dos órgãos abdominais e, algumas vezes, ocorre falha desde a região torácica ventral, ocasionando também a saída dos órgãos torácicos, sendo chamada de toracosquise. Neste último, além da fenda no tórax, há também uma falha na formação dos elementos do arcabouço da caixa torácica (Jericó et al., 2015).

Diversas hipóteses têm sido aventadas para explicar a patogênese da gastrosquise. Todas envolvem um defeito ou rompimento da parede abdominal no período embrionário com subsequente herniação do intestino fetal. Dentre elas pode-se citar a falha do mesoderma para formar a parede abdominal, ruptura do âmnio ao redor do anel umbilical, involução anormal da veia umbilical, levando a uma fragilidade da parede abdominal e ruptura da artéria vitelina direita com subsequente lesão da parede abdominal fetal (Salihu et al., 2004).

Diante do exposto, o presente trabalho tem o objetivo de relatar um caso de malformação congênita, caracterizada por gastrosquise e toracosquise, em neonato canino.

\section{Relato de caso}

Uma cadela com seis anos de idade, sem raça definida, com peso de $18,8 \mathrm{~kg}$, foi admitida para atendimento em um Hospital Veterinário, com queixa clínica de apresentar uma secreção gelatinosa na região da vulva há duas semanas. Durante a anamnese, a proprietária relatou que o animal estava gestante e que tinha feito a aplicação de anticoncepcional dois dias após a cadela ter copulado com um cão. Ao exame físico, observou-se aumento de volume da região abdominal e das glândulas mamárias, secreção vaginal consistente com trabalho de parto. Os demais parâmetros do exame físico estavam dentro dos padrões de normalidade para a espécie.

Foram solicitados exames hematológicos de hemograma e bioquímicos (ureia, creatinina, ALT alanina aminotransferase e FA - fosfatase alcalina), os quais não apresentaram alterações. Também foi solicitado o exame ultrassonográfico abdominal da paciente, no qual visibilizou-se a presença de três fetos viáveis, sem sofrimento fetal e idade gestacional de aproximadamente de 60 dias. Assim, o animal foi encaminhado para o procedimento cirúrgico de cesariana seguida de ovariohisterectomia.

A indução anestésica feita com propofol (Diprivan ${ }^{\circledR}$, Astrazeneca, São Paulo - SP, Brasil) na dose 4 $\mathrm{mg} / \mathrm{kg}$, por via intravenosa (IV). Ato contínuo, o animal foi conectado a um sistema com reinalação (valvular infantil) para administração de isofluorano (Forane ${ }^{\circledR}$, Abbott, Rio de Janeiro - RJ, Brasil) diluído em 100\% de oxigênio. Depois, realizou-se o bloqueio anestésico regional, por meio de anestesia epidural $(0,25 \mathrm{~mL} / \mathrm{kg})$ utilizando lidocaína $2 \%$ sem vasoconstritor e tramadol $5 \%$ na dose de $0,1 \mathrm{mg} / \mathrm{kg}$, ambos na mesma seringa. Trinta minutos antes do procedimento cirúrgico, fez-se antibioticoterapia profilática com ampicilina na dose $20 \mathrm{mg} / \mathrm{kg}$ IV.

A terapia pós-operatória consistiu em meloxicam na dose de $0,1 \mathrm{mg} / \mathrm{kg}$ a cada 24 horas por três dias, dipirona na dose $25 \mathrm{mg} / \mathrm{kg}$ a cada oito horas por cinco dias e, para a limpeza da ferida cirúrgica prescreveu-se clorexidine spray a cada 12 horas por dez dias. As suturas cutâneas foram removidas no décimo dia do período pós-operatório. 
Durante a cesariana, observou-se a presença de três fetos vivos. E no decorrer dos cuidados neonatais, foi notado que apenas um filhote neonatal apresentava uma alteração de continuidade da parede abdominal e torácica, sendo diagnosticado como gastroquise e toracosquise.

O neonato apresentava pelos distribuídos por todo o corpo, ausência de defeitos craniofaciais, sem alterações em membros torácicos e pélvicos, comprimento aproximado de $15,8 \mathrm{~cm}, 10 \mathrm{~cm}$ de circunferência abdominal e 9,2 cm de circunferência torácica, pesando aproximadamente 400 gramas. Observou-se um defeito na parede abdominal (músculo reto do abdômen) e torácica (músculos peitorais superficiais e profundo) de aproximadamente $4 \mathrm{~cm}$ de comprimento, permitindo a saída dos órgãos abdominais como intestino, estômago, omento, fígado, baço. Durante a exploração da cavidade torácica, notou-se a ausência de esterno, um defeito no diafragma, com exposição de pulmão e coração. Todos os órgãos estavam hiperêmicos, hemorrágicos, cobertos por fibrina e colágeno.

Diante disso, optou-se pela eutanásia do neonato, seguindo as normas de ética do Colégio Brasileiro de Experimentação Animal dos recém-nascidos, sendo realizada com propofol na dose de $5 \mathrm{mg} / \mathrm{kg}$ por via intravenosa e, após o animal entrar em plano anestésico, foi aplicado cloreto de potássio na dose de $100 \mathrm{mg} / \mathrm{kg}$ também por via intravenosa.

\section{Discussão}

As alterações congênitas possuem etiologias de origem genéticas ou hereditárias, de origem iatrogênica (tratamentos médicos administrados à mãe durante a gestação), nutricionais por desequilíbrios alimentares (excesso de vitaminas ou proteínas) e virais (herpes-vírus ou parvovírus tipo I) (Prats et al., 2005; Sorribas, 2007). Alteração congênita desse relato foi ocasionada pela aplicação do anticoncepcional à base de medroxiprogesterona, pois sabe-se que a gastrosquise é passível de ser induzida em laboratório por uma variedade de agentes como, por exemplo, antagonistas de estrogênio em cães (Curry et al., 2000). As toxinas, os teratógenos e os agentes químicos podem originar morte fetal, aborto ou diversos efeitos congênitos (Johnston et al., 2001; Traas, 2008). Alguns relatos de gastrosquise foram observados em felinos (Miranda et al., 2013; Siqueira et al., 2013). Siqueira et al. (2003), e ainda são escassas informações sobre gastrosquise e toracosquise na espécie canina.

Miranda et al. (2013) relataram um caso três neonatos felinos, um deles apresentava alteração congênita craniofacial, e os outros dois, gastrosquise. Já no relato em questão, dois neonatos caninos apresentavam-se sem alterações e somente um apresentava malformação de gastrosquise e toracosquise. Siqueira et al. (2013) trataram um felino que apresentava gastrosquise, e após duas intervenções cirúrgicas, o animal veio a óbito por dilatação gástrica, entretanto não pôde concluir se o óbito foi devido a complicações da malformação ou resultado de casualidade.

Neste relato, além da gastrosquise em que os órgãos da cavidade abdominal se desenvolveram fora do corpo e estavam em contato com o líquido amniótico, o neonato também apresentava alterações macroscópicas de toracosquise, com fenda em tórax, por defeito no fechamento ventral do embrião e da formação do arcabouço e hérnia diafragmática corroborando com o que foi relatado por Jericó et al. (2015); entretanto, sem alterações de membros torácicos e membros pélvicos.

A correção cirúrgica apresenta como complicações a desproporção entre vísceras e cavidade abdominal, favorecendo o comprometimento das funções do trato gastrintestinal ou respiratório e predispondo a infecções, sendo assim, o neonato terá baixa expectativa de vida. Devido ao grande defeito anatômico observado no neonato do presente estudo e as escassas informações na literatura de como proceder com essas malformações congênitas na medicina veterinária bem como a falha no tratamento de suporte neonatal, optou-se pela eutanásia do animal.

\section{Conclusão}

As malformações congênitas de gastrosquise e toracosquise possuem tratamento limitado, sugerindo a necessidade de estudos sobre o desenvolvimento embrionário, a eficiência de técnicas cirúrgicas aplicáveis e tratamento de suporte neonatal adequado, a fim de garantir um bom prognóstico para os pacientes portadores. 


\section{Referências bibliográficas}

Curry, J. I., McKinney, P., Thornton, J. G., \& Stringer, M. D. (2000). The aetiology of gastroschisis. BJOG: An International Journal of Obstetrics \& Gynaecology, 107(11), 1339-1346. https://doi.org/10.1111/j.1471-0528.2000.tb11645.x.

Jericó, M. M., Kogika, M. M., \& Andrade Neto, J. P. (2015). Tratado de medicina interna de cães e gatos. Guanabara Koogan.

Johnston, S. D., Olson, M. V, Johnston, P. S. S. D., Kustritz, M. V. R., \& Olson, P. N. S. (2001). Canine and feline theriogenology. WB Saunders.

Little, S. (2011). Feline pediatrics: how to treat the small and the sick. Compendium: Continuing Education For Veterinarians, 33, 1-6.

Miranda, D. F. H., Azevedo Júnior, J. W. C., Ferraz, M. S., Pessoa, G. T., Bezerra, D. O., Fortes, E. A. M., \& Conde Júnior, A. M. (2013). Malformações congênitas em neonatos felinos: relato de caso. PUBVET, 7, 259-311. https://doi.org/10.22256/pubvet.v7n4.1503.

Prats, A., Prats, A., García, F., Dumon, C., Martí, S., \& Coll, V. (2005). Neonatologia e pediatria canina e felina. Interbook.

Salihu, H. M., Emusu, D., Aliyu, Z. Y., Pierre-Louis, B. J., Druschel, C. M., \& Kirby, R. S. (2004). Mode of delivery and neonatal survival of infants with isolated gastroschisis. Obstetrics \& Gynecology, 104(4), 678-683. https://doi.org/10.1097/01.AOG.0000139513.93115.81.

Siqueira, E. G. M., Quitzan, J. G., \& Brandão, C. V. S. (2013). Gastrósquise em neonato felino e cirurgia reparadora. Anais Do III Simpósio de Neonatologia Veterinária, 22.

Sorribas, C. E. (2007). Atlas de neonatologia y pediatria en caninos. Inter-medica.

Traas, A. M. (2008). Resuscitation of canine and feline neonates. Theriogenology, 70(3), 343-348.

Histórico do artigo:

Recebido: 16 de março de 2021

Aprovado: 29 de abri de 2021
Licenciamento: Este artigo é publicado na modalidade Acesso Aberto sob a licença Creative Commons Atribuição 4.0 (CC-BY 4.0), a qual permite uso irrestrito, distribuição, reprodução em qualquer meio, desde que o autor e a fonte sejam devidamente creditados. 